\title{
Injectivity and the Law of Demand*
}

\author{
Roy Allen \\ Department of Economics \\ University of Western Ontario \\ rallen46@uwo.ca
}

August 19, 2019

\begin{abstract}
Establishing that a demand mapping is injective is core first step for a variety of methodologies. When a version of the law of demand holds, global injectivity can be checked by seeing whether the demand mapping is constant over any line segments. When we add the assumption of differentiability, we obtain necessary and sufficient conditions for injectivity that generalize classical Gale and Nikaido [1965] conditions for quasi-definite Jacobians.
\end{abstract}

*I thank Nail Kashaev, Salvador Navarro, John Rehbeck, and David Rivers for helpful comments. 


\section{Introduction}

A variety of recently developed methods require, as a first step, that a demand mapping be injective. Examples include work on endogeneity with market level data (Berry 1994, Berry, Levinsohn, and Pakes [1995], Berry and Haile 2009], Chiappori and Komunjer [2009], Berrv and Haile [2014]); simultaneous equations models (Matzkin [2008], Matzkin [2015], Berrv and Haile [2018]); multidimensional heterogeneity in a consumer setting (Blundell et al. [2017]); index models (Ahn. Ichimura. Powell, and Ruud [2017]); and nonparametric analysis in trade (Adao et al. [2017]). 1

The applicability of these methods depends on whether the demand mapping is injective. This paper studies injectivity using a shape restriction that allows complementarity: the law of demand.

Definition 1. $Q: U \subseteq \mathbb{R}^{K} \rightarrow \mathbb{R}^{K}$ satisfies the law of demand if for each $u, \tilde{u} \in U$,

$$
(Q(u)-Q(\tilde{u})) \cdot(u-\tilde{u}) \geqslant 0
$$

Many models imply a version of the law of demand, both in the standard consumer problem and outside it. In the standard consumer problem, $u$ is the negative of the price vector. Quasilinear preferences imply the law of demand 2 Hildenbrand 1983] provides conditions under which the law of demand holds in the aggregate, even if it does not hold at the individual level 3 Outside the standard consumer problem, the discrete choice additive random utility model (McFadden [1981]) also satisfies the law of demand. In that model one may interpret $u_{k}$ as the deterministic utility index for alternative $k$ and $Q(u)$ as a vector of choice probabilities.

Directly checking whether a demand mapping is injective is nontrivial. This paper provides necessary and sufficient conditions for injectivity that can simplify this task. The simplest condition states that when demand is continuous and the domain of

\footnotetext{
${ }^{1}$ Chesher and Rosen 2017] and Bonnet et al. 2017] take an alternative approach, working with inverse images that may be multivalued.

${ }^{2}$ Fosgerau et al. 2018] provide an injectivity result for a demand system that allows complementarity. They consider quasilinear preferences and so their demand system fits into the setup of this paper.

${ }^{3}$ Hildenbrand [1983] also provides sufficient conditions that ensure a strict law of demand $(Q(u)-$ $Q(\tilde{u})) \cdot(u-\tilde{u})>0$ for $u \neq \tilde{u}$, which clearly implies injectivity.
} 
demand is convex, global injectivity of $Q$ can be checked by checking whether $Q$ is constant over line segments. This implies that global and local injectivity are equivalent.

The main result of this paper is a nondifferentiable counterpart to the classical injectivity results of Gale and Nikaido [1965] for functions with weakly quasi-definite Jacobians 4 When I specialize the main result by assuming the demand mapping $Q$ is differentiable, I establish a generalization of Gale and Nikaido [1965], Theorem 6w. While Gale and Nikaido [1965] impose invertibility of the Jacobian of $Q$ as a sufficient condition for global injectivity, I provide a necessary and sufficient condition for local (and global) injectivity in terms of certain directional derivatives.

Berry, Gandhi, and Haile 2013] have recently shown that demand mappings that satisfy a "connected substitutes" property are injective. This connected substitutes condition applies to a number of existing models, including models of market shares based on a discrete choice foundation (e.g. Berry and Haile [2014]), but may not apply when there is complementarity 5 This paper complements their analysis by studying injectivity using a shape restriction that allows complementarity without a reparametrization.

The injectivity results of this paper exploit the fact that when $Q$ is continuous and satisfies the law of demand, the inverse image of any quantity is a convex set. This is a classical result in monotone operator theory 6 To my knowledge, this important property has not been exploited for studying injectivity in the econometrics literature, yet it has several implications that I describe further in the paper. The closest precedent appears to be in the study of uniqueness of general equilibrium, where several conditions are known to yield convexity of equilibria (e.g. Arrow and Hurwicz [1958], Arrow and Hurwicz [1960], and the discussion in Mas-Colell [1991]).

\footnotetext{
${ }^{4}$ To be clear, this paper only overlaps when the Jacobian is weakly quasi-definite, not just a $P$ matrix as in Theorem 4 in Gale and Nikaido [1965].

5 Berry et al. 2013] show in several examples that certain models with complements may be reparametrized to fit into their setup. See also Brown and Matzkin [1998] and Beckert and Blundell [2008] for injectivity results that allow complementarity between goods.

${ }^{6}$ See e.g. Rockafellar and Wets [2009].
} 


\section{Characterization of Injectivity}

This section presents the main results, which provide necessary and sufficient conditions for a demand mapping to be injective. We use the following assumption, which allows us to reduce checking global injectivity to checking local conditions.

Assumption 1. $Q: U \subseteq \mathbb{R}^{K} \rightarrow \mathbb{R}^{K}$ satisfies the law of demand, is continuous, and $U$ is open and convex.

Recall that a set $U$ is convex if for $u, \tilde{u} \in U$ and any scalar $\alpha \in[0,1]$, it follows that $\alpha u+(1-\alpha) \tilde{u} \in U$. An important implication of this assumption is that inverse images $Q^{-1}(u)$ are convex, which I formalize below. As discussed in the Introduction, this is a classical result in monotone operator theory.

Lemma 1. Let Assumption 1 hold. Then for each $y \in \mathbb{R}^{K}$,

$$
Q^{-1}(y)=\{u \in U \mid Q(u)=y\}
$$

is convex.

Proof. If the domain is $U=\mathbb{R}^{K}$, this is a textbook result, e.g. Rockafellar and Wets 2009], p. 536. When $U \neq \mathbb{R}^{K}$, this is covered by Kassay, Pintea, and Szenkovits [2009], Theorem 3.5.

When $K>1$, continuity cannot be dropped without alternative structure, as the following example illustrates.

Example 1. Let $K=2, U=\mathbb{R}^{2}$, and $A=\left\{u \in \mathbb{R}^{2} \mid u_{1}+u_{2}>0\right.$ or $\left.u_{1}=u_{2}=0\right\}$. Let $Q(u)=(1\{u \in A\}, 1\{u \in A\})$, where $1\{u \in A\}$ is an indicator function for whether $u \in A$. To show the law of demand is satisfied, note that if $u, \tilde{u}$ are either both in $A$ or both in its complement $A^{c}, Q$ does not vary and clearly satisfies the law of demand. Consider then $u \in A, \tilde{u} \in A^{c}$. Then we have

$$
(Q(u)-Q(\tilde{u}))^{\prime}(u-\tilde{u})=\left(u_{1}-\tilde{u}_{1}\right)+\left(u_{2}-\tilde{u}_{2}\right) \geqslant 0
$$

Nonetheless, $Q^{-1}(0,0)=A^{c}$ is not convex, since both points $(-1,1),(1,-1)$ are in $A^{c}$, yet their convex combination $(0,0)$ is not.

Using Lemma 1, we obtain the following list of conditions that are equivalent to global 
injectivity of $Q$.

Proposition 1. Let Assumption 11 hold. Then the following are equivalent:

i. $Q$ is injective, i.e. for each $y \in \mathbb{R}^{K}$ there is at most one $u \in U$ such that $Q(u)=y$.

ii. $Q$ is locally injective, i.e. for each $u \in U$ there is a neighborhood $H \subseteq \mathbb{R}^{K}$ of $u$ such that the restriction of $Q$ to $H$ is injective.

iii. The only line segments in $U$ along which $Q$ is constant are singleton points.

Proof. Clearly, (i) $\Longrightarrow$ (ii) $\Longrightarrow$ (iii). That (iii) $\Longrightarrow$ (i) follows from Lemma1 1 and the definition of convexity. Indeed, if $Q(u)=Q(\tilde{u})$, then the set $Q^{-1}(Q(u))$ is convex and must contain $u$ and $\tilde{u}$. In particular, $Q$ is constant on any line segment joining $u$ and $\tilde{u}$. By assumption, this is only possible if $u=\tilde{u}$.

Local injectivity always implies condition (iii), but in general the reverse is not true. An example of a multivariate function that satisfies (iii) but is not locally injective is $Q\left(u_{1}, u_{2}\right)=\left(u_{1}^{3}-u_{2}, u_{1}^{3}-u_{2}\right)$. The equivalence of (i) and (iii) is the most powerful part of Proposition 1, since part (iii) is often easy to check. In addition, we can leverage this equivalence to relax the domain restrictions. We formalize this as follows.

Corollary 1. Let Assumption 1 hold. Let $Q_{H}: H \subseteq U \rightarrow \mathbb{R}^{K}$ denote the restriction of $Q$ to the set $H$. If $H$ is open or convex, any of the injectivity conditions of Proposition 1 are equivalent when applied to the function $Q_{H}$.

Proof. It is clear that (i) $\Longrightarrow$ (ii) $\Longrightarrow$ (iii). It remains to show that (iii) $\Longrightarrow$ (i).

Let $Q_{H}(u)=Q_{H}(\tilde{u})$ for $u, \tilde{u} \in H$. Let $T \subseteq U$ be the line segment from $u$ to $\tilde{u}$. From Lemma 1, we conclude that $Q^{-1}\left(Q_{H}(u)\right)$ contains $T$, and hence $Q_{H}^{-1}\left(Q_{H}(u)\right)$ contains $T \cap H$. In particular, $Q_{H}$ is constant over $T \cap H$.

Suppose for the purpose of contradiction that $u \neq \tilde{u}$. If $H$ is either open or convex, the set $T \cap H$ contains two distinct line segments that are not points, beginning at $u$ and $\tilde{u}$, respectively. Since we assumed (iii) holds, we reach a contradiction since $Q_{H}$ is constant over $T \cap H$, and thus is constant over these line segments.

Note that this proposition assumes $Q$ satisfies the law of demand over the entire set $U$. This assumption may be satisfied by appealing to economic theory. It allows one 
to show injectivity for the restriction $Q_{H}$ for a set $H$ that is either open or convex. The primary reason one may be interested in such restrictions is that one may only have information on $Q$ over a certain region of utility indices (such as $H$ ).

The proof of Corollary 1 establishes the following additional result.

Corollary 2. Let Assumption 1 hold. Let $Q_{H}: H \subseteq U \rightarrow \mathbb{R}^{K}$ denote the restriction of $Q$ to the set $H$. If $H$ is open or convex, the following are equivalent for arbitrary $u \in H$ :

i. For any $u, \tilde{u} \in H$ with $u \neq \tilde{u}, Q_{H}(u) \neq Q_{H}(\tilde{u})$, i.e. the inverse image $Q_{H}^{-1}\left(Q_{H}(u)\right)$ is a singleton.

ii. $Q_{H}$ is locally injective at $u$, i.e. there is a neighborhood $N \subseteq H$ of $u$ such that the restriction of $Q_{H}$ to $N$ is injective.

iii. The only line segment in $H$ that contains $u$ and and over which $Q_{H}$ is constant is the point $u$.

The equivalence of (i) and (ii) shows that to check whether an inverse image is a singleton, it is enough to check features of the mapping $Q_{H}$ that are local to a single u. Importantly, this differs from Proposition 1 and Corollary 1, which instead study how local injectivity holding for each $u$ implies global injectivity. Thus, Corollary 2 further highlights the sense in which injectivity can be reduced to a local condition. It also conceptually differs from classical papers such as Gale and Nikaido [1965], which focus on when a local condition holding everywhere implies global injectivity.

Finally, to see that these equivalences do not hold in general, consider $H=U=\mathbb{R}$ and $Q(u)=u^{2}$, which is continuous yet violates the law of demand. Then $Q_{H}$ is locally injective at $u=1$, but $Q_{H}^{-1}\left(Q_{H}(1)\right)=\{-1,1\}$.

\section{Relationship to Gale and Nikaido [1965]}

In this section I describe how the local-to-global injectivity result of Proposition [1] may be seen as a nondifferentiable version of a classical result due to Gale and Nikaido [1965]. In drawing this relationship, I present a new result complementing their results 
for weakly quasi-definite Jacobians, which drops the requirement that the function have a convex domain.

I now add the assumption that $Q$ is differentiable.

Assumption 2. The function $Q: U \subseteq \mathbb{R}^{K} \rightarrow \mathbb{R}^{K}$ is differentiable, where $U$ is an open, convex set.

To relate to Gale and Nikaido [1965], I introduce some definitions.

Definition 2. $A K \times K$ matrix $B$ is positive semi-definite if $\lambda^{\prime} B \lambda \geqslant 0$ for every $\lambda \in \mathbb{R}^{K}$. If $\lambda^{\prime} B \lambda>0$ for every nonzero $\lambda$, then $B$ is positive definite.

Definition 3. A $K \times K$ matrix $B$ is weakly quasi-definite if $\left(B+B^{\prime}\right) / 2$ is positive semi-definite. If $\left(B+B^{\prime}\right) / 2$ is positive definite, then $B$ is quasi-definite.

The following result connects the law of demand and quasi-definiteness of Jacobians.

Lemma 2. Let Assumption 2 hold. The function $Q$ satisfies the law of demand if and only if its Jacobian is everywhere weakly quasi-definite.

Proof. See e.g. Parthasarathy [2006], p. 92.

With this lemma and the previous results, we obtain a generalization of Gale and Nikaido [1965], Theorem 6w.

Proposition 2. Let Assumption 2 hold and suppose $Q$ satisfies the law of demand. Let $H \subseteq U$ be an open set and let $Q_{H}$ denote the restriction of $Q$ to $H$. Then $Q_{H}$ is injective if its Jacobian is everywhere invertible.

Proof. From Corollary 1 we see it is enough to establish local injectivity of $Q_{H}$. Since the Jacobian of $Q_{H}$ is everywhere invertible, $Q_{H}$ is locally injective by Proposition 3 because its directional derivatives are never zero 7

Gale and Nikaido [1965] prove this result when $H=U$, i.e. over convex domains 8 The link between the law of demand and results in Gale and Nikaido [1965] has previously been noted (Kassay, Pintea, and Szenkovits [2009], László [2016]). Proposi-

\footnotetext{
${ }^{7}$ Note that if we had assumed $Q$ is continuously differentiable, we could apply the classical inverse function theorem to establish local injectivity. We have not assumed the derivative is continuous, and hence we use an alternative technique.

${ }^{8}$ Theorem 6 in that paper requires instead that $U$ be convex and drops openness, but instead also requires that the Jacobian be positive quasi-definite (not just weakly quasi-definite, which is implied by the law of demand).
} 
tion 2 is new to my knowledge, and handles the important case of checking injectivity over a nonconvex set.

\section{Relaxing the Jacobian Condition}

The previous section uses the invertibility of the Jacobian of $Q$ as a sufficient condition for local injectivity of $Q$. Invertibility of the Jacobian is not necessary for local injectivity, as illustrated for $K=1$ by $Q(u)=u^{3}$, since the derivative is 0 at 0 .

Obtaining necessary and sufficient conditions for local injectivity in terms of derivatives is nontrivial in general. For $Q$ satisfying the law of demand, by Proposition 1(iii), however, checking global or local injectivity is equivalent to checking whether $Q$ is constant over any line segment that is not a point. One may write this condition in terms of certain directional derivatives. To that end, define the directional derivative of $Q$ at $u$ in direction $v$, denoted $Q^{\prime}(u, v)$, by

$$
\left|\lim _{\lambda \downarrow 0} \frac{Q(u+\lambda v)-Q(u)}{\lambda}-Q^{\prime}(u, v)\right|=0
$$

whenever this limit exists.

Proposition 3. Let Assumption 2 hold and suppose $Q$ satisfies the law of demand. The following are equivalent for arbitrary $u \in U$ :

i. For any $\tilde{u} \in U$ with $u \neq \tilde{u}, Q(u) \neq Q(\tilde{u})$.

ii. $Q$ is locally injective at $u$.

iii. The only line segment in $U$ that contains $u$ and and over which $Q$ is constant is the point $u$.

$i v$. There are no nonzero vectors $v \in \mathbb{R}^{K}$ such that for all $\lambda \in[0,1]$ satisfying $u+\lambda v \in$ $U, Q^{\prime}(u+\lambda v, v)$ is the zero vector.

Proof. Since $Q$ is differentiable, it is continuous. The equivalence between (i)-(iii) follows from Corollary 2. Equivalence between (iii) and (iv) follows from the mean value theorem. 
Note that if the Jacobian of $Q$ is invertible at $u \neq 0$, then $Q^{\prime}(u, v)$ cannot be the zero vector for any nonzero $v$, because directional derivatives satisfy $J(u) v=Q^{\prime}(u, v)$, where $J(u)$ is the Jacobian of $Q$ at $u$. Thus if $Q$ has an everywhere invertible Jacobian, condition (iii) is satisfied for each $u \in U$. More generally, suppose that failures of invertibility of the Jacobian of $Q$ only occur on an isolated set of points. Then clearly, condition (iii) is satisfied for each $u \in U$.

Recall from Proposition 1, global injectivity of $Q$ is equivalent to local injectivity for each $u \in U$. Thus, global injectivity of $Q$ is also equivalent to conditions (ii) or (iii) of Proposition 3 holding for each $u \in U$.

By combining Lemma 2 and Proposition 3 , we obtain a generalization of Gale and Nikaido [1965], Theorem 6. This generalization drops the assumption that the Jacobian of $Q$ is everywhere invertible.

Corollary 3. Let Assumption Q hold and assume the Jacobian of $Q$ is everywhere weakly quasi-definite. The following are equivalent:

i. $Q$ is injective.

ii. For each $u \in U$, any of the equivalent conditions in Proposition 9 holds.

\section{Complements, Substitutes, and the Law of De- mand}

Berry, Gandhi, and Haile [2013] have recently shown that a "connected substitutes" condition implies global injectivity. The present paper shows that a version of the law of demand, which allows complementarity, also suffices. This approach is not nested in and does not nest that of Berry, Gandhi, and Haile [2013].

The setup of Berry, Gandhi, and Haile [2013] imposes the following properties on the demand mapping $Q: U \subseteq \mathbb{R}^{K} \rightarrow \mathbb{R}^{K}$ :

i. (Strict Own-Good Monotonicity) Let $k$ be arbitrary. For each $u, \tilde{u} \in U$ such that $u_{k}>\tilde{u}_{k}$ and $u_{j}=\tilde{u}_{j}$ for $j \neq k$, it follows that

$$
Q_{k}(u)>Q_{k}(\tilde{u})
$$


ii. (Weak Substitutability) Let $k$ be arbitrary. For each $u, \tilde{u} \in U$ such that $u_{k}>\tilde{u}_{k}$ and $u_{j}=\tilde{u}_{j}$ for $j \neq k$, it follows that for all $\ell \neq k$,

$$
Q_{\ell}(u) \leqslant Q_{\ell}(\tilde{u})
$$

Condition (i) states demand increases in its own utility shifter. Condition (ii) states that if a utility shifter for good $k$ increases, then all other demands weakly decrease. Condition (i), except with a weak inequality, follows whenever $Q$ satisfies the law of demand, but Condition (ii) does not.

Berry, Gandhi, and Haile [2013] impose a "connected substitutes" condition, which directly assumes weak substitutability and implies strict own-good monotonicity (see Remark 1 in Berry, Gandhi, and Haile [2013]). For brevity, I omit a formal statement, and instead describe a key implication of their assumption: for arbitrary $u, \tilde{u} \in U$,

$$
Q(u) \geqslant Q(\tilde{u}) \Longrightarrow u \geqslant \tilde{u}
$$

where $\geqslant$ denotes the usual partial order in $\mathbb{R}^{K}$, i.e. $u \geqslant \tilde{u}$ if and only if $u_{k} \geqslant \tilde{u}_{k}$ for each $k$. This shape restriction is called inverse isotonicity, and clearly implies that $Q$ is injective 9 This property is essential for establishing injectivity using the approach of Berry, Gandhi, and Haile [2013].

As discussed previously, the methods of this paper are distinct from those of Berry, Gandhi, and Haile [2013]. I provide two examples showing the distinction between inverse isotonicity and the law of demand. First, I show that the law of demand does not imply inverse isotonicity.

Example 2. Consider a linear demand system $Q(u)=A u$, where

$$
A=\left[\begin{array}{ll}
2 & 1 \\
1 & 2
\end{array}\right] .
$$

The matrix $A$ is symmetric and satisfies row-diagonal dominance (e.g. for each row, the diagonal $|2|$ exceeds the sum of the off-diagonal $|1|)$, which are well-known conditions that ensures the matrix $\left(A+A^{\prime}\right) / 2$ is positive semi-definite. Thus, the law

\footnotetext{
${ }^{9}$ Appendix $\mathrm{A}$ describes a characterization of inverse isotonicity in this setting using results in Moré and Rheinboldt [1973].
} 
of demand is established from Lemma Q2. This demand mapping violates weak substitutability because the off-diagonals of $A$ are positive. In addition, it violates inverse isotonicity. To see this, consider the two vectors $u=(0,0), \tilde{u}=(2,-1)$. Then $Q(u)=(0,0), Q(\tilde{u})=(3,0)$ and so $Q(\tilde{u}) \geqslant Q(u)$, but we do not have $\tilde{u} \geqslant u$.

The following example illustrates that inverse isotonicity does not imply the law of demand.

Example 3. Now consider a demand system

$$
Q(u)=\left[\begin{array}{cc}
20 & -10 \\
-1 & 2
\end{array}\right]\left[\begin{array}{l}
u_{1}^{3} \\
u_{2}^{3}
\end{array}\right]
$$

The function $Q$ satisfies the connected substitutes property of Berry, Gandhi, and Haile [2013], hence inverse isotonicity. It does not satisfy the law of demand. To see this, consider $u=(0,0)$ and $\tilde{u}=(1,2)$. One obtains $Q(u)=(0,0)$ and $Q(\tilde{u})=(-60,7)$. Thus,

$$
(Q(\tilde{u})-Q(u))^{\prime}(\tilde{u}-u)=(-60,7)^{\prime}(1,2)=-46<0 .
$$

In this example, the law of demand fails because the substitution effect outweighs the own-good effect.

To shed further light on the distinction between inverse isotonicity and the law of demand, it is helpful to note that the law of demand is not an ordinal property. This is illustrated in Example 3 by considering the strictly increasing function $f(v)=v^{1 / 3}$. Consider the transformed mapping

$$
\tilde{Q}(u)=Q\left(\left(f\left(u_{1}\right), f\left(u_{2}\right)\right)\right)=\left[\begin{array}{cc}
20 & -10 \\
-1 & 2
\end{array}\right]\left[\begin{array}{l}
u_{1} \\
u_{2}
\end{array}\right] .
$$

The mapping $\tilde{Q}$ satisfies the law of demand even though the original mapping $Q$ in Example 3 violates the law of demand 10 Further analysis of the law of demand and a change of variables is covered in Section 7 .

Finally, it is clear that strict own-good monotonicity, weak substitability, and inverse isotonicity are all ordinal properties in the following sense: they hold for some mapping $Q(u)$ if and only if they hold for $\tilde{Q}(u)=Q(f(u))$ where $f(u)=$

\footnotetext{
${ }^{10}$ This follows because the symmetrized matrix of coefficients in $\tilde{Q}$ satisfies diagonal dominance.
} 
$\left(f_{1}\left(u_{1}\right), \ldots, f_{K}\left(u_{K}\right)\right)$, and each $f_{k}$ is strictly increasing.

\section{Quasilinear Utility}

In the standard consumer problem, quasilinear utility is a well-known class of preferences that implies the law of demand. I provide an injectivity result that exploits additional structure of this model.

Suppose an individual maximizes a utility function of the form

$$
y_{0}+C\left(y_{1}, \ldots, y_{k}\right)
$$

with budget constraint $\sum_{k=0}^{K} p_{k} y_{k} \leqslant M$. Suppose $p_{0}$ does not vary and is normalized to 1. Under local nonsatiation and allowing negative quantities of $y_{0}$ (or sufficiently high income), the maximization problem is equivalent to choosing quantities to maximize

$$
-\sum_{k=1}^{K} p_{k} y_{k}+C\left(y_{1}, \ldots, y_{k}\right)
$$

where $y_{0}=M-\sum_{k=1}^{K} p_{k} y_{k}$ has been substituted out. Thus, if the maximizer is unique we have

$$
Q(u)=\underset{y \in \mathbb{R}^{K}}{\operatorname{argmax}} \sum_{k=1}^{K} u_{k} y_{k}+C(y),
$$

where $u_{k}=-p_{k}$; more generally, we may take $Q(u)$ to be an element of the argmax correspondence.

To see that $Q$ satisfies the law of demand, consider the necessary condition for maximization

$$
\sum_{k=1}^{K} u_{k} Q_{k}(u)+C(Q(u)) \geqslant \sum_{k=1}^{K} u_{k} Q_{k}(\tilde{u})+C(Q(\tilde{u})) 11
$$

An analogous inequality holds with $u$ and $\tilde{u}$ reversed. Summing up the two analogous inequalities and rearranging establishes that $Q$ satisfies the law of demand.

\footnotetext{
${ }^{11}$ If there are multiple maximizers, this inequality holds for any maximizers. In particular, a law of demand holds for arbitrary selectors from the argmax correspondence.
} 
Quasilinear models imply (weakly) more than the law of demand (Brown and Calsamiglia [2007]). The additional structure of quasilinear models yields the following result. Note that the domain $U$ need not be convex.

Lemma 3 (cf. Rockafellar [1970], Theorems 23.5 and 25.1). Let $Q: U \subseteq \mathbb{R}^{K} \rightarrow \mathbb{R}^{K}$ satisfy

$$
Q(u) \in \underset{y \in \mathbb{R}^{K}}{\operatorname{argmax}} \sum_{k=1}^{K} u_{k} y_{k}+C(y)
$$

where $U$ is open. If $C: \mathbb{R}^{K} \rightarrow \mathbb{R} \cup\{+\infty\}$ is concave, upper semi-continuous,12 and finite at some point, then the following are equivalent for arbitrary $u \in U$ :

i. $C$ is differentiable at $Q(u)$.

ii. There is no $\tilde{u} \in U$ such that $u \neq \tilde{u}$ and $Q(u)=Q(\tilde{u})$.

This result directly follows from Rockafellar [1970] and so the proof is omitted. A corollary of this lemma is that if $C$ is everywhere differentiable (and the other conditions are met), then $Q$ is globally invertible. A version of this result has been used in Allen and Rehbeck [2019]; I include this result for completeness, to illustrate how additional structure allows us to further specialize the results, and because the quasilinear structure is widely used.

\subsection{Relation to Brown and Matzkin [1998]}

A structure that shares a mathematical relationship with quasilinear utility has been studied in Brown and Matzkin [1998]. Suppose now that there is a budget constraint but the demand is not quasilinear. Formally, the consumer solves the problem

$$
\begin{array}{r}
\max _{\left(y_{0}, y\right) \in \mathbb{R}_{+}^{K+1}} \sum_{k=1}^{K} u_{k} y_{k}+C\left(y_{0}, y\right) \quad \text { s.t. } \\
\sum_{k=1}^{K} p_{k} y_{k}+y_{0} \leqslant I,
\end{array}
$$

\footnotetext{
${ }^{12} \mathrm{~A}$ function $f: \mathbb{R}^{K} \rightarrow \mathbb{R} \cup\{+\infty\}$ is upper semi-continuous if $\{y \mid f(y) \geqslant \alpha\}$ is closed for each $\alpha$.
} 
where $I$ is income. Brown and Matzkin [1998] study this model with the interpretation that $u$ is a vector of exogenous unobservables 13 Blundell et al. [2017] study a generalization that is not covered by our setup.

Note that fixing prices and income, this problem differs from the setup of Lemma 3 only because of the budget constraint. However, under local nonsatiation the constraint is satisfied with equality and so the problem reduces to

$$
\max _{y \in \mathbb{R}_{+}^{K}} \sum_{k=1}^{K} u_{k} y_{k}+C\left(I-\sum_{k=1}^{K} p_{k} y_{k}, y\right) .
$$

Injectivity of this demand system is then covered by Lemma 3. In particular, fixing prices and income, differentiability of the mapping $\tilde{C}(y)=C\left(I-\sum_{k=1}^{K} p_{k} y_{k}, y\right)$ and injectivity are equivalent under certain conditions, as formalized in Lemma 3$]^{14} \mathrm{Im}-$ portantly, while this argument can provide sharp conditions relating injectivity and differentiability, it does not establish smoothness of the inverse. Indeed, differentiability of $\tilde{C}$ does not rule out multiple maximizers, and so the demand mapping need not even be continuous. Brown and Matzkin [1998] provide additional conditions that ensure injectivity and smoothness.

\subsection{Discrete Choice and Aggregation}

Many models outside of the consumer problem that have additively separable unobservable heterogeneity also share the structure of the quasilinear utility model. In particular, they imply a version of the law of demand in utility indices that need not involve price. For the additive random utility model, this has been recognized at least since the seminal work of McFadden [1981]. Other examples that share this structure are the discrete choice bundles model of Gentzkow [2007], the matching model of Fox. Yang. and Hsu 2018], and a model of decisions under uncertainty considered in Agarwal and Somaini [2018]; see Allen and Rehbeck [2019] for details.

\footnotetext{
${ }^{13}$ Their presentation is slightly different since they consider also a term $u_{0} y_{0}$ in the utility and then normalize $u_{0}=1$. With this normalization, this term can be absorbed into $C\left(y_{0}, y\right)$.

${ }^{14} \mathrm{~A}$ related change of variables argument has appeared in Allen and Rehbeck [2019] to study a discrete choice problem.
} 
Example 4 (Additive Random Utility Models (McFadden [1981])). Let

$$
v_{j}=u_{j}+\varepsilon_{j}
$$

denote the latent utility for alternative $j$. Treat $\varepsilon=\left(\varepsilon_{1}, \ldots, \varepsilon_{K}\right)$ as a random variable and $u$ as a constant. Normalize the latent utility of the outside good $(j=0)$ to 0 and assume there are $K$ inside goods. Suppose the individual chooses an alternative that maximizes latent utility and let $\tilde{D}(u, \varepsilon) \in\{0,1\}^{K}$ be a vector of indicators denoting denoting which, if any, of the inside goods $(j>0)$ is chosen. Then by similar arguments as in the quasilinear utility example, necessary conditions for optimality imply

$$
(\tilde{D}(u, \varepsilon)-\tilde{D}(\tilde{u}, \varepsilon)) \cdot(u-\tilde{u}) \geqslant 0 .
$$

Moreover, letting $Q(u)=\mathbb{E}[\tilde{D}(u, \varepsilon)]$ where the expectation is over $\varepsilon$, we have

$$
(Q(u)-Q(\tilde{u})) \cdot(u-\tilde{u}) \geqslant 0
$$

In this example, $Q(u)$ is the vector of probabilities for choosing each of the $K$ inside goods 15 This example illustrates two principles. First, the law of demand is preserved under expectations. In particular, the law of demand holding at the individual level implies it holds at the aggregate level 16 Second, injectivity results may be used for aggregate data even when injectivity fails at the individual level. Note that for fixed $\varepsilon$, the function $\tilde{D}(\cdot, \varepsilon)$ cannot be injective whenever $U$ has more than $K+1$ points. However, taking expectations can serve to smooth out discreteness and restore injectivity. Whether $Q$ is injective depends on the distribution of $\varepsilon$ (Norets and Takahashi [2013]; see also Azevedo, Weyl, and White [2013]).

\footnotetext{
${ }^{15}$ In this paper I treat $u$ as a fixed parameter. If $u$ is treated as an observable random variable, then as long as $u$ is independent of $\varepsilon$ (and some technical conditions are met), $Q(u)$ is the conditional probability of choosing each alternative, conditional on the shifters $u$.

${ }^{16}$ See Shi et al. 2018] for a related application of this principle for discrete choice panel data.
} 


\section{Law of Demand with a Change of Variables}

In some models the law of demand does not hold, but holds after a change of variables. We can adapt Proposition 1 to such settings when the change of variables is sufficiently well-behaved. I formalize that the law of demand holds after a change of variables as follows.

Assumption 3. $\tilde{Q}(u)=Q(f(u))$, where $Q: U \subseteq \mathbb{R}^{K} \rightarrow \mathbb{R}^{K}$ satisfies the law of demand and is continuous, $f: T \subseteq \mathbb{R}^{K} \rightarrow U \subseteq \mathbb{R}^{K}$, and $U$ and $T$ are open and convex.

When $f$ is a homeomorphism, i.e. a continuous function with a continuous inverse, we still obtain that local injectivity implies global injectivity.

Proposition 4. Let Assumption 3 hold with $f$ a homeomorphism. Then the following are equivalent:

i. $\tilde{Q}$ is injective.

ii. $\tilde{Q}$ is locally injective.

Proof. Clearly (i) $\Longrightarrow$ (ii), and so we wish to show (ii) $\Longrightarrow$ (i). By Lemma 1, the set $Q^{-1}(Q(f(u)))$ is convex, hence connected, for each $u \in U$. Since $f^{-1}$ is continuous, its image of the connected set $Q^{-1}(Q(f(u)))$ is connected 17 Hence, $\tilde{Q}^{-1}(\tilde{Q}(u))=$ $f^{-1}\left(Q^{-1}(Q(f(u)))\right)$ is connected.

By the assumption of local injectivity of $\tilde{Q}$, the set $\tilde{Q}^{-1}(\tilde{Q}(u))$ consists of isolated points. That is, each $\tilde{u} \in \tilde{Q}^{-1}(\tilde{Q}(u))$ has a neighborhood $H$ such that $H \cap \tilde{Q}^{-1}(\tilde{Q}(u))=$ $\tilde{u}$. Since $\tilde{Q}^{-1}(\tilde{Q}(u))$ is connected, nonempty and consists of isolated points, it can have exactly one point. Since this is true for arbitrary $u$, we obtain part (i).

An example of a homeomorphism is $f(u)=\left(f_{1}\left(u_{1}\right), \ldots, f_{K}\left(u_{K}\right)\right)$, where each $f_{k}$ is strictly increasing and continuous and $T$ is rectangular (i.e. the Cartesian product of intervals). Note that we no longer conclude that checking local injectivity of $\tilde{Q}$ is equivalent to checking whether it is constant on line segments.

\footnotetext{
${ }^{17}$ Recall a set is connected if it cannot be partitioned into two disjoint nonempty sets that are open in the relative topology. Convex sets are clearly connected.
} 
We can obtain a sharper result with alternative structure on $f$. Say that a mapping $f: \mathbb{R}^{K} \rightarrow \mathbb{R}^{K}$ is affine if it may be written $f(u)=A u+b$ for some $K \times K$ matrix $A$ and vector $b \in \mathbb{R}^{K}$. Affine mappings need not satisfy the law of demand 18 Nonetheless, affine mappings have the important property that for a convex set $B \subseteq \mathbb{R}^{K}, f^{-1}(B)$ is convex. By leveraging Lemma 1, this leads to the following result.

Proposition 5. Let Assumption 3 hold for affine $f$. Then for each $y \in \mathbb{R}^{K}$,

$$
\tilde{Q}^{-1}(y)=\{u \in T \mid \tilde{Q}(u)=y\}
$$

is convex. In particular, the following are equivalent:

i. $\tilde{Q}$ is injective.

ii. $\tilde{Q}$ is locally injective.

iii. The only line segments in $U$ along which $Q$ is constant are points.

Proof. The set $Q^{-1}(\tilde{Q}(u))$ is convex. Thus the set $\tilde{Q}^{-1}(\tilde{Q}(u))=f^{-1}\left(Q^{-1}(\tilde{Q}(u))\right)$ is convex because $f$ is affine. The result is then analogous to Proposition 1 .

\section{Discussion}

This paper leverages a classical result in monotone operator theory to provide simple necessary and sufficient conditions to check when a demand mapping is injective. Specifically, for continuous demand mappings that satisfy the law of demand and that are defined over an open convex domain, local injectivity and global injectivity are equivalent. In addition, injectivity can be checked by seeing if the demand mapping is constant over any line segments that are not points. I describe the relationship to a classical result of Gale and Nikaido [1965] for quasi-definite Jacobians, providing necessary and sufficient conditions for global injectivity in terms of directional derivatives. Finally, I show that the law of demand is not nested in and does not nest the "connected substitutes" condition of Berry et al. [2013].

\footnotetext{
${ }^{18}$ They do precisely when the symmetrized matrix $\left(A+A^{\prime}\right) / 2$ is positive semi-definite (e.g. Rockafellar [1970], p. 240), where $A^{\prime}$ denotes the transpose of $A$. This can be seen by writing $(f(u)-f(\tilde{u}))^{\prime}(u-\tilde{u})=(u-\tilde{u})^{\prime} A^{\prime}(u-\tilde{u})$ and recalling the definition of the law of demand.
} 


\section{Appendix A Inverse Isotonicity and Substitution}

To keep the paper self-contained, I provide a primitive condition that ensures the demand mapping satisfies inverse isotonicity.

Lemma 4 (Moré and Rheinboldt [1973]). Let $Q: U \subseteq \mathbb{R}^{K} \rightarrow \mathbb{R}^{K}$, where $U$ is a Cartesian product. In addition, assume $Q$ satisfies strict own-good monotonicity and weak substitutability. The following are equivalent:

1. Q satisfies inverse isotonicity.

2. $Q$ is a P-function, i.e. for $u \neq \tilde{u}$, there is some $k$ such that

$$
\left(Q_{k}(u)-Q_{k}(\tilde{u})\right)\left(u_{k}-\tilde{u}_{k}\right)>0 \text {. }
$$

We note that while Moré and Rheinboldt [1973] prove this result for $U$ a rectangle (i.e. a Cartesian product of intervals), their proofs go through without modification when $U$ is an arbitrary Cartesian product. $P$-functions are closely related to functions whose Jacobians are $P$-matrices, whose injectivity properties are studied in Gale and Nikaido [1965]. See Moré and Rheinboldt [1973] for more details.

From this result we conclude that because the connected substitutes assumption of Berry, Gandhi, and Haile 2013] implies inverse isotonicity, the demand mapping in their setup is a $P$-function. This can be deduced from their Lemma 3, which states that under their assumptions, if $u \neq \tilde{u}$ and $\mathcal{I}=\left\{k \mid u_{k}>\tilde{u}_{k}\right\}$ is nonempty, then

$$
\sum_{k \in \mathcal{I}} Q_{k}(u)>\sum_{k \in \mathcal{I}} Q_{k}(\tilde{u})
$$

This implies that there must be some $k \in \mathcal{I}$ such that $\left(Q_{k}(\tilde{u})-Q_{k}(u)\right)\left(u_{k}-\tilde{u}_{k}\right)>0$, i.e. $Q$ must be a $P$-function 19

\footnotetext{
${ }^{19}$ Note that if $\mathcal{I}$ is empty we can repeat the argument with $u$ and $\tilde{u}$ interchanged.
} 


\section{References}

Rodrigo Adao, Arnaud Costinot, and Dave Donaldson. Nonparametric counterfactual predictions in neoclassical models of international trade. American Economic Review, 107(3):633-89, 2017.

Nikhil Agarwal and Paulo Somaini. Demand analysis using strategic reports: An application to a school choice mechanism. Econometrica, 86(2):391-444, 2018.

Hyungtaik Ahn, Hidehiko Ichimura, James L Powell, and Paul A Ruud. Simple estimators for invertible index models. Journal of Business 85 Economic Statistics, (just-accepted), 2017.

Roy Allen and John Rehbeck. Identification with additively separable heterogeneity. Econometrica, 8(3):1021-1054, 2019.

Kenneth J Arrow and Leonid Hurwicz. On the stability of the competitive equilibrium, i. Econometrica: Journal of the Econometric Society, pages 522-552, 1958.

Kenneth J Arrow and Leonid Hurwicz. Some remarks on the equilibria of economic systems. Econometrica: Journal of the Econometric Society, pages 640-646, 1960.

Eduardo M Azevedo, E Glen Weyl, and Alexander White. Walrasian equilibrium in large, quasilinear markets. Theoretical Economics, 8(2):281-290, 2013.

Walter Beckert and Richard Blundell. Heterogeneity and the non-parametric analysis of consumer choice: conditions for invertibility. The Review of Economic Studies, 75(4):1069-1080, 2008.

Steven Berry, James Levinsohn, and Ariel Pakes. Automobile prices in market equilibrium. Econometrica: Journal of the Econometric Society, pages 841-890, 1995.

Steven Berry, Amit Gandhi, and Philip Haile. Connected substitutes and invertibility of demand. Econometrica, 81(5):2087-2111, 2013.

Steven T Berry. Estimating discrete-choice models of product differentiation. The RAND Journal of Economics, pages 242-262, 1994.

Steven T Berry and Philip A Haile. Nonparametric identification of multinomial 
choice demand models with heterogeneous consumers. Technical report, National Bureau of Economic Research, 2009.

Steven T Berry and Philip A Haile. Identification in differentiated products markets using market level data. Econometrica, 82(5):1749-1797, 2014.

Steven T Berry and Philip A Haile. Identification of nonparametric simultaneous equations models with a residual index structure. Econometrica, 86(1):289-315, 2018.

Richard Blundell, Dennis Kristensen, Rosa Matzkin, et al. Individual counterfactuals with multidimensional unobserved heterogeneity. Technical report, Centre for Microdata Methods and Practice, Institute for Fiscal Studies, 2017.

Odran Bonnet, Alfred Galichon, Keith O'Hara, and Matthew Shum. Yogurts choose consumers? identification of random utility models via two-sided matching. 2017.

Donald J Brown and Caterina Calsamiglia. The nonparametric approach to applied welfare analysis. Economic Theory, 31(1):183-188, 2007.

Donald J Brown and Rosa Liliana Matzkin. Estimation of nonparametric functions in simultaneous equations models, with an application to consumer demand. Citeseer, 1998.

Andrew Chesher and Adam M Rosen. Generalized instrumental variable models. Econometrica, 85(3):959-989, 2017.

P Chiappori and Ivana Komunjer. On the nonparametric identification of multiple choice models. Manuscript, Columbia University, 2009.

Mogens Fosgerau, Andre de Palma, and Julien Monardo. Demand models for differentiated products with complementarity and substitutability. 2018.

Jeremy T Fox, Chenyu Yang, and David H Hsu. Unobserved heterogeneity in matching games. Journal of Political Economy, 126(4):000-000, 2018.

David Gale and Hukukane Nikaido. The jacobian matrix and global univalence of mappings. Mathematische Annalen, 159(2):81-93, 1965. 
Matthew Gentzkow. Valuing new goods in a model with complementarity: Online newspapers. American Economic Review, 97(3):713-744, 2007.

Werner Hildenbrand. On the" law of demand". Econometrica: Journal of the Econometric Society, pages 997-1019, 1983.

Gábor Kassay, Cornel Pintea, and Ferenc Szenkovits. On convexity of preimages of monotone operators. Taiwanese Journal of Mathematics, pages 675-686, 2009.

Szilárd László. On injectivity of a class of monotone operators with some univalency consequences. Mediterranean Journal of Mathematics, 13(2):729-744, 2016.

Andreu Mas-Colell. On the uniqueness of equilibrium once again. In William A Barnett, Bernard Cornet, Claude D'Aspremont, Jean Gabszewicz, and Andreu Mas-Colell, editors, Equilibrium Theory and Applications: Proceedings of the Sixth International Symposium in Economic Theory and Econometrics, chapter 12, pages 275-296. Cambridge University Press Cambridge, 1991.

Rosa L Matzkin. Identification in nonparametric simultaneous equations models. Econometrica, 76(5):945-978, 2008.

Rosa L Matzkin. Estimation of nonparametric models with simultaneity. Econometrica, 83(1):1-66, 2015.

Daniel McFadden. Econometric models of probabilistic choice. In Charles F. Manski and Daniel McFadden, editors, Structural Analysis of Discrete Data with Econometric Applications. Mit Press Cambridge, MA, 1981.

J Moré and Werner Rheinboldt. On p-and s-functions and related classes of ndimensional nonlinear mappings. Linear Algebra and its Applications, 6:45-68, 1973.

Andriy Norets and Satoru Takahashi. On the surjectivity of the mapping between utilities and choice probabilities. Quantitative Economics, 4(1):149-155, 2013.

Thiruvenkatachari Parthasarathy. On global univalence theorems, volume 977 of Lecture Notes in Mathematics. Springer, 2006.

R Tyrrell Rockafellar. Convex Analysis. Princeton University Press, 1970. 
R Tyrrell Rockafellar and Roger J-B Wets. Variational analysis, volume 317. Springer Science \& Business Media, 2009.

Xiaoxia Shi, Matthew Shum, and Wei Song. Estimating semi-parametric panel multinomial choice models using cyclic monotonicity. Econometrica, 86(2):737-761, 2018. 